\title{
High-resolution computed tomography in clinical T1 No MO adenocarcinoma of the lung
}

Valerie W. Rusch, MD

See related article on page 278 .
From the Memorial Sloan-Kettering Cancer Center, New York, NY.

Received for publication Jan 15, 2002; accepted for publication Feb 22, 2002.

Address for reprints: Valerie W. Rusch, MD, Thoracic Service, Department of Surgery, Memorial Sloan-Kettering Cancer Center, 1275 York Ave, New York, NY 10021 (E-mail: ruschv@mskcc.org).

J Thorac Cardiovasc Surg 2002;124:221-2

Copyright (C) 2002 by The American Association for Thoracic Surgery

0022-5223/2002 \$35.00+0 $\quad \mathbf{1 2 / 1 / 1 2 4 6 6 7}$

doi: $10.1067 / \mathrm{mtc} .2002 .124667$

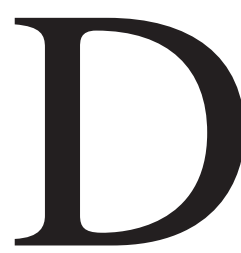

espite much controversy surrounding the effectiveness of lowdose helical computed tomographic (CT) scanning for the early detection of lung cancer, ${ }^{1}$ the use of CT for this purpose is already widespread and is rapidly changing clinical practice. Radiologists and thoracic surgeons are confronted with the need to distinguish tiny benign pulmonary lesions from very early lung cancers and with the challenge of determining when biopsy or resection is appropriate. However, there are no absolute criteria for identifying very early lung cancers on CT or for determining preoperatively the clinical and biologic behavior of an individual tumor.

The widespread use of CT scanning is also challenging our approach to pulmonary resection. Lobectomy has been the standard form of resection for most lung cancers, based in part on a randomized trial by the Lung Cancer Study Group showing that lesser resections were associated with a significantly higher risk of local recurrence. ${ }^{2}$ Now the increasing diagnosis of very early lung cancers-usually defined as T1a tumors that are $2 \mathrm{~cm}$ or less in size-is leading thoracic surgeons to question whether some of these cancers could be managed appropriately by a lesser resection. Approximately $10 \%$ to $20 \%$ of very early cancers are associated with lymph node metastases. However, the remainder have a more indolent biologic behavior. The risk of nodal metastases and of subsequent systemic disease is partly but not entirely linked to the size and location of the primary tumor. The ability to distinguish which of these small tumors will have either an aggressive or indolent biologic behavior is critically important.

Defining which of these very early lung cancers can be managed with lesser pulmonary resections is important not only to decrease local recurrence, minimize operative morbidity, and preserve lung function but also because these patients survive long enough to be at risk for a second or even a third lung cancer. The risk of second primary lung cancers is roughly $2.5 \%$ per year. Patients who survive 5 or more years after their initial lung cancer resection therefore face a significant cumulative risk of second primary tumors. The greater the extent of the first resection, the more limited are the surgical options for subsequent resections.

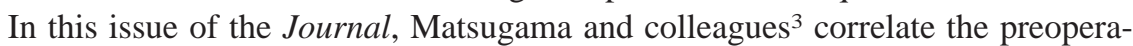
tive high-resolution CT scan (HRCT) findings with the pathologic features and outcomes of 96 patients who underwent resection of clinical stage Ia non-small cell lung cancer. They find that the proportion of ground-glass opacity (GGO), defined as a hazy increase in lung attenuation without obscuring the underlying vascular marking, on HRCT is directly related to tumor histology, which in turn is linked to the risk of nodal metastases and long-term survival. Specifically, all of the cancers measuring $2 \mathrm{~cm}$ or less and for which the proportion of GGO to the whole tumor shadow on CT was $50 \%$ or greater were node-negative bronchioloalveolar carcinomas (BACs). None of these tumors recurred after resection. The authors suggest that the proportion of GGO on preoperative HRCT correlates with the pathologic features of these very early cancers, predicts tumor aggressiveness and might be useful in selecting patients for limited pulmonary resection.

As the authors point out in the "Discussion" section of their article, this analysis is part of an emerging body of work correlating findings on imaging studies with the pathologic features and clinical outcome of very early lung cancers. ${ }^{4-7}$ In particular, 
BACs that are characterized by a lepidic growth pattern along the alveoli without areas of invasion present as areas of GGO on CT, show little or no uptake on FDG-positron emission tomographic scan, and usually have an indolent behavior. ${ }^{8,9}$ Because areas of BAC are often seen at the periphery of invasive adenocarcinomas, it is hypothesized that they might represent the preinvasive form of lung adenocarcinoma. Along with previous reports, the current article by Matsuguma and colleagues ${ }^{3}$ certainly suggests that limited pulmonary resection might be a correct approach to the management of small BACs. However, much additional investigation is needed in this area. Reliable methods are needed for characterizing and quantifying GGO on HRCT. The findings in the current study need to be confirmed in larger, multicenter studies. The CT characteristics and the risk of nodal metastases for small lung cancers that are predominantly invasive carcinomas rather than BACs need to be fully defined. Very importantly, the molecular features of these different entities (ie, BAC, BAC with focal invasion, and adenocarcinoma with BAC) need to be identified and correlated with both imaging findings and with clinical outcome.

Understanding the biology of these very early lung cancers could also provide insight into the behavior of somewhat more advanced tumors. For instance, the genomic or protein abnormalities identified in very small invasive tumors that have lymph node metastasis could provide clues to the risk of systemic disease in larger tumors. This area of investigation has become sufficiently important that it was the subject of a 2-day meeting at the National Cancer Institute in June $2001 .{ }^{10}$ At that meeting, it was agreed that large multicenter studies are needed to define the imaging characteristics, clinical and pathologic features, and molecular abnormalities of very early lung cancers. Plans for such studies are underway in several groups, including the American College of Surgeons Oncology Group. The results of these studies will probably significantly alter our approach to the diagnosis and management of very early lung cancers, which are becoming an increasingly important part of thoracic surgical practice.

\section{References}

1. Patz EF Jr, Goodman PC, Bepler G. Screening for lung cancer. $N$ Engl $J$ Med. 2000;343:1627-33.

2. Ginsberg RJ, Rubinstein LV. Randomized trial of lobectomy versus limited resection for T1N0 non-small cell lung cancer. Lung Cancer Study Group. Ann Thorac Surg. 1995;60:615-22.

3. Matsuguma H, Yokoi K, Anraku M, Kondo T, Kamiyama Y, Mori K, Tominaga K, Tsuura Y, Honjo S. Proportion of ground-glass opacity on high-resolution computed tomography in clinical T1 N0 M0 adenocarcinoma of the lung: a predictor of lymph node metastasis. $J$ Thorac Cardiovasc Surg. 2002;124:278-84.

4. Ichinose Y, Yano T, Yokoyama H, Inoue T, Asoh H, Katsuda Y. The correlation between tumor size and lymphatic vessel invasion in resected peripheral stage I non-small-cell lung cancer. A potential risk of limited resection. J Thorac Cardiovasc Surg. 1994;108:684-6.

5. Takizawa $\mathrm{T}$, Terashima $\mathrm{M}$, Koike $\mathrm{T}$, Watanabe $\mathrm{T}$, Kurita $\mathrm{Y}$, Yokoyama A, et al. Lymph node metastasis in small peripheral adenocarcinoma of the lung. J Thorac Cardiovasc Surg. 1998;116:27680 .

6. Asamura H, Nakayama H, Kondo H, Tsuchiya R, Shimosato Y, Naruke T. Lymph node involvement, recurrence, and prognosis in resected small, peripheral, non-small cell lung carcinomas: Are these carcinomas candidates for video-assisted lobectomy? J Thorac Cardiovasc Surg. 1996;111:1125-34

7. Noguchi M, Morikawa A, Kawasaki M, Matsuno Y, Yamada T, Hirohashi S, et al. Small adenocarcinoma of the lung. Histologic characteristics and prognosis. Cancer. 1995;75:2844-52.

8. Kuriyama K, Seto M, Kasugai T, Higashiyama M, Kido S, Sawai Y, et al. Ground-glass opacity on thin-section CT: value in differentiating subtypes of adenocarcinoma of the lung. AJR Am J Roentgenol. 1999;173:465-9.

9. Kodama K, Higashiyama M, Yokouchi H, Takami K, Kuriyama K, Mano M, et al. Prognostic value of ground-glass opacity found in small lung adenocarcinoma on high-resolution CT scanning. Lung Cancer. 2001;33:17-25.

10. Lung Cancer State of the Science Meeting: Diagnosis and Therapy of Screening-Detected Lung Cancer; Bethesda, Md, 2001, June 19-20. Meeting report available at: http://www.webtie.org/SOTS/html/ Lung\%20Home.htm. 